\title{
A CFD DESIGN OF ENGINEERED SURFACE FOR TRIBOLOGICAL PERFORMANCE IMPROVEMENTS IN HYDRAULIC PUMPS
}

\author{
Paolo Casoli $^{1 *}$, Fabio Scolari ${ }^{1}$, Carlo Rossi ${ }^{2}$, Manuel Rigosi ${ }^{2}$ \\ ${ }^{1}$ University of Parma, Parco Area delle Scienze 181/A, 43124 Parma, Italy \\ ${ }^{2}$ Casappa S.p.A., Via Balestrieri 1, 43044, Lemignano di Colecchio PR, Italy \\ * Corresponding author: Tel.: +390521 905868; E-mail address: paolo.casoli@unipr.it
}

\begin{abstract}
In the present paper the preliminary results of the potentialities that surface texturing has in improving the coupling of lubricated surfaces in relative motion is presented. This kind surface engineering requires careful design of the geometry to obtain relevant improvements; therefore, it is useful to study in detail the behavior of the fluid confined between the coupled surfaces by means of CFD analysis. The purpose of this research is to study the effect of dimples created on one of the two coupled surfaces and to observe the variation of tribological properties as their principal design parameters vary, such as dimple shape, size and spatial distribution. Furthermore, simulations have been carried out with different sliding velocities and fluid temperatures to analyze the effects that these variables have on the tribological performance of the textured surface. The simulations also consider the presence of cavitation and the influence of this phenomenon on the overall behavior of the textured surface is evaluated.
\end{abstract}

Keywords: laser surface texturing, CFD simulation

\section{INTRODUCTION}

Lubricated couplings between surfaces in relative motion are very common in hydraulic machines and new solutions able to improve their performance could lead to extend their working range.

The adoption of textured surfaces can lead to different benefits and improve the tribological properties of the surface coupling [1].

The geometric effects permit to obtain a greater hydrodynamic bearing capacity and a lower friction coefficient due also to a smaller contact area, which would allow to reduce the overall wear. Furthermore a "hollow" effect could occur with the function of collecting extraneous particles and contaminants as well as creating a reserve of oil is useful to avoid the breaking of lubricating film.

Accurate design of the surface geometry is a tough challenge because of the high number of variables that influence the final behavior of the coupling. Furthermore, the characteristics of the dimple profile strongly depend on the type of contact that occurs between the two facing surfaces, as well as the operating conditions [2].
Some researchers have studied these solutions applied to parallel thrust bearings, demonstrating that modifying the coupled surfaces in relative motion could lead to improved hydrodynamic lubrication [3, 4]. Other studies investigate the effect of surface texturing realized on circumferential seals proving that the dimples are able to generate a hydrodynamic force that could reduce the seal friction and wear $[5,6]$.

Many studies in this field have been carried out by means of finite difference method to numerically solve the Reynolds lubrication equation [6-8].

In this research in order to evaluate the influence of the geometrical characteristics of the dimples and the coupled surfaces, such as the gap and the relative sliding speed between the two coupled surfaces, in addition to the change in viscosity of the fluid, several three-dimensional CFD simulations were performed, the analysis were carried out using ANSYS ${ }^{\circledR}$ CFX to solve the Navier-Stokes equations and the energy equation.

The simulations were carried out considering cavitation phenomenon which could occur under some operating conditions and is able to modify 
the coupling performance, even leading to negative effects.

This paper reports a preliminary study of the potentials of a textured surface. The final aim of this research is to apply textured surfaces on some components inside hydraulic pumps. Both in external gear pumps and in axial piston pumps there are numerous lubricated coupled surfaces that can become critical beyond certain operating conditions. The outlook is to extend the working range of these machines using the benefits offered by engineered surfaces.

\section{CFD MODELLING OF TEXTURED SURFACES}

\subsection{Fluid model}

The fluid has been modeled within the various CFD analysis performed is an ISO VG-46 hydraulic oil with the characteristics listed in the Table 1.

Table 1: Fluid parameters.

\begin{tabular}{ll}
\hline Density & $850 \mathrm{~kg} / \mathrm{m}^{3}$ \\
Viscosity $\left(40^{\circ} \mathrm{C}\right)$ & $46 \mathrm{~mm}^{2} / \mathrm{s}$ \\
Molar mass & $240 \mathrm{~kg} / \mathrm{mol}$ \\
Specific heat capacity & $1950 \mathrm{~J} /(\mathrm{kg} \mathrm{K})$ \\
Relative saturation pressure & $-0.7 \mathrm{bar}$ \\
\hline
\end{tabular}

The analyses were performed considering the cavitation phenomenon, using the Rayleigh Plesset model [9]. In order to correctly model this phenomenon the definition of the physical and thermodynamic properties of the fluid is essential. Since the reference hydraulic oil is a complex mixture of heavy hydrocarbons, to simplify the properties definition the fluid has been assumed mono component considering the heptadecane $\mathrm{C}_{17} \mathrm{H}_{35}$. The vapor phase has been modelled as a real gas adopting a cubic equation of state, according to the Soave Redlich Kwong model [10]. All the simulations were carried out setting the fluid temperature equal to $40^{\circ} \mathrm{C}$.

\subsection{Single dimple}

As reference geometry a square-shaped dimple has been adopted. In Figure 1 a schematic representation of the domain and the dimple position is shown, with the main geometric features indicated.

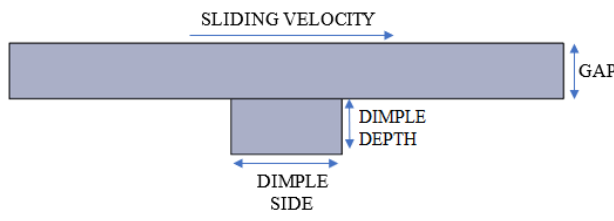

Figure 1: Graphic representation of the dimple with the main geometric features indicated.

The domain dimensions have been chosen in order to allow the pressure gradient due to the presence of the dimple to reach the zero value in both the $\mathrm{x}$ and $\mathrm{y}$ directions, as shown in the pressure contour in Figure 2. The reference domain is $500 \mu \mathrm{m}$ long in the $\mathrm{x}$ direction and 300 $\mu \mathrm{m}$ along $\mathrm{y}$. The reference value of the sliding velocity is assumed to be $5 \mathrm{~m} / \mathrm{s}$ which is a typical value in many hydraulic applications.

The gap is in the order of micron, since it is a common value that characterize the lubricated couplings in hydraulic machines.

The boundary conditions are set as open boundary with a relative pressure of 0 bar.

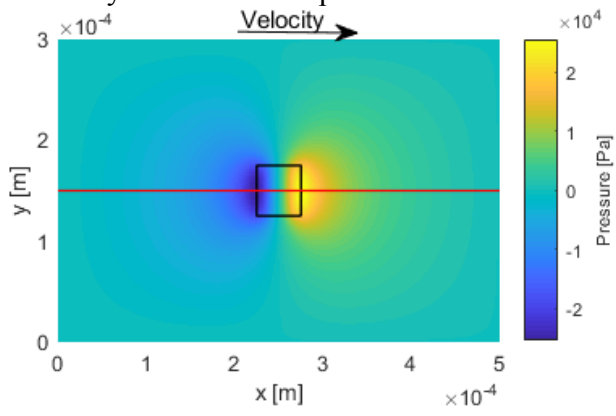

Figure 2: Pressure contour of a square dimple with side $50 \mu \mathrm{m}$ and depth $10 \mu \mathrm{m}$. Gap $10 \mu \mathrm{m}$, sliding velocity $5 \mathrm{~m} / \mathrm{s}$

Several simulations have been performed varying the geometrical features of the dimple and of the gap as well as the viscosity of the fluid and the sliding velocity. Figure 3 reports the results concerning geometrical features, the pressure profile is calculated along the dimple median line on the moving plane (indicated by the red line in Figure 2) and it oscillates symmetrically around the mean value. The mean value is the reference relative pressure imposed as boundary condition.

It follows that the sustaining force generated is always equal to zero, regardless of the pressure conditions at the boundary, the working temperature (i.e. the viscosity of the fluid) and the relative sliding speed between the two surfaces. 


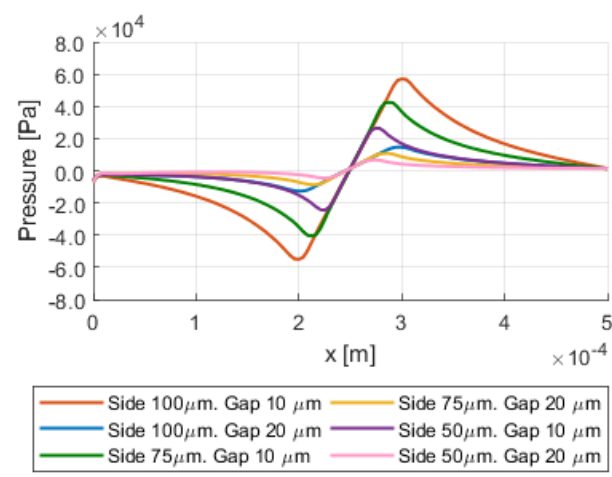

Figure 3: Pressure profile perturbation generated by a square dimple with different size and different value of the gap. Dimple depth $10 \mu \mathrm{m}$, sliding velocity $5 \mathrm{~m} / \mathrm{s}$

One aspect to be observed is the influence of the size of the dimple. Dimples characterized by large dimensions in the moving direction generate a pressure profile characterized by a bigger amplitude than smaller ones, but still symmetrical. Besides the reduction of the gap leads to similar effects, increasing the amplitude of the pressure oscillation.

These conclusions can also be extended to other dimple geometries that have a symmetrical structure, such as a rectangular or circular geometry; as a matter of fact in all these configurations the divergent zone is exactly the same as the convergent one, leading to a symmetrical pressure profile that do not create any bearing capacity.

However, there could be some operating conditions where, due to the onset of cavitation phenomenon, some sort of bearing action arises. As shown in Figure 3, both decreasing the gap and increasing the dimple size, bring to a higher amplitude of the pressure profile; as a consequence the minimum pressure value of the profile will be lower.

In the Figure 4 it is possible to notice that the same square dimple produces a pressure profile of greater amplitude as the clearance decreases; for those cases where, in the depression area, the pressure drops down below the fluid saturation limit, cavitation arises. As a consequence, the pressure profile is not symmetric anymore and some sort of bearing effect is provided.

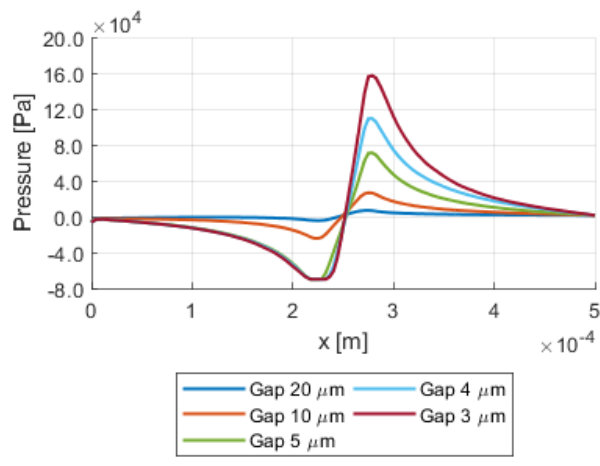

Figure 4: Pressure profile of a square dimple with side of $50 \mu \mathrm{m}$ and depth $10 \mu \mathrm{m}$ for different value of the gap. Sliding velocity $5 \mathrm{~m} / \mathrm{s}$

In Figure 5 the load capacity generated by dimples of different dimensions is shown; the effect is amplified if the gap clearance is reduced. The values are divided by a reference value for confidential reasons. The force is the integral of the pressure calculated on the entire domain surface and the trend is more than linear as far as the gap thickness is decreased.

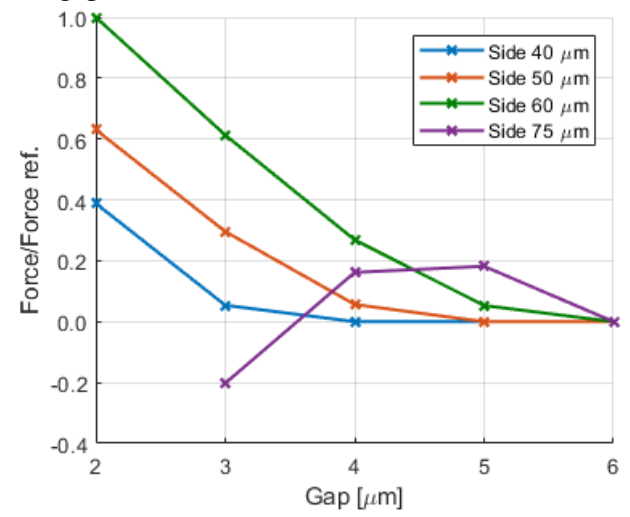

Figure 5: Bearing capacity. Influence of the gap for different size of the dimple. Dimple depth $10 \mu \mathrm{m}$, sliding velocity $5 \mathrm{~m} / \mathrm{s}$.

The increasing of the bearing capacity with the reduction of the gap could be a useful effect to compensate any approaching trend, hence preventing the condition of direct contact between the two surfaces.

From Figure 5 emerges that larger dimples offer more benefits in term of load capacity; however a limit exists, beyond which the trend is opposite. Furthermore, the largest dimples are 
characterized by greater cavitation phenomena with a wider area at saturation pressure. In Figure 6 the pressure contour on the moving plane of a large square dimple is reported, while in Figure 7 its own pressure profile, calculated along the central red line, is shown. The overall integral of the pressure on the surface is negative; in this case, worsening the bearing performance of the coupling.

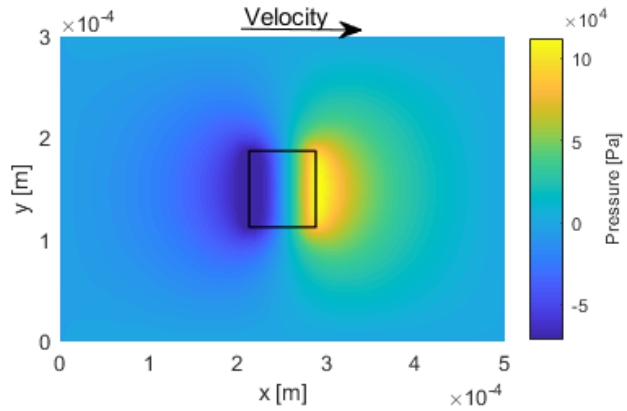

Figure 6: Pressure contour of a square dimple with side $75 \mu \mathrm{m}$ and depth $10 \mu \mathrm{m}$. Gap $5 \mu \mathrm{m}$, sliding velocity $5 \mathrm{~m} / \mathrm{s}$

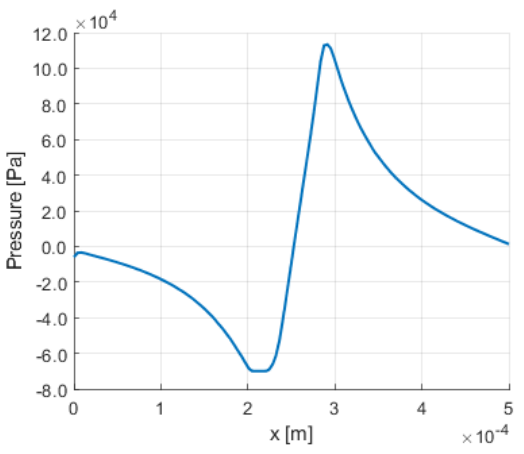

Figure 7: Pressure profile of a large square dimple with side $75 \mu \mathrm{m}$ and depth $10 \mu \mathrm{m}$. Gap 5 $\mu \mathrm{m}$, sliding velocity $5 \mathrm{~m} / \mathrm{s}$

In all the simulations presented the boundary conditions were set 0 bar, this value affect the minimum and maximum values of the pressure profile. Setting the pressure boundary at higher value the minimum pressure value can remain over the saturation pressure and cavitation is avoided. The generation of the bearing capacity is due exclusively to the onset of cavitation, therefore by modifying the pressure boundary condition, greater than 0 bar, cavitation does not occur and the pressure profile remains symmetrical without generating any force.

\subsection{Influence of operating conditions and dimples depth}

According to the lubrication equation and the Reynolds theory, Eq. 1, the dependence of the pressure on the fluid viscosity is linear. The viscosity of the fluid is related to the working temperature and decreases while it rises.

Therefore, the pressure profile near the dimple is amplified with lower fluid temperature (higher viscosity).

$$
\frac{\partial}{\partial x}\left(h^{3} \frac{\partial p}{\partial x}\right)=6 U \eta \frac{d h}{d x}
$$

According to Eq. 1, the dependence of the pressure on the sliding speed between the two coupled surfaces is also linear; changing the fluid viscosity or the sliding velocity of a certain extent, the pressure profile will be scaled in the same proportion. In Figure 8 it is possible to appreciate the variations in the pressure profile as a function of both the viscosity of the fluid and the sliding velocity, while in Table 2 the amplitude value of the pressure profile is reported as confirmation of the linear relation described above.

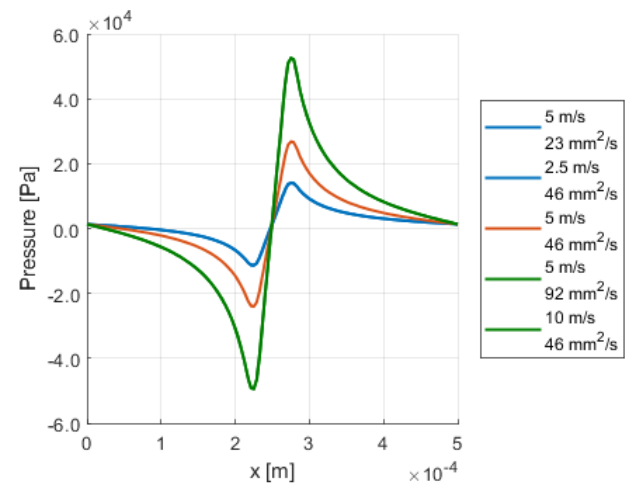

Figure 8: Effect of viscosity and velocity.

Table 2: Amplitude of the pressure profile.

\begin{tabular}{cc}
\hline Parameters & Amplitude [Pa] \\
\hline $5 \mathrm{~m} / \mathrm{s}$ & \\
$23 \mathrm{~mm}^{2} / \mathrm{s}$ & $1.27 \mathrm{E}+04$ \\
$2.5 \mathrm{~m} / \mathrm{s}$ & \\
$46 \mathrm{~mm}^{2} / \mathrm{s}$ & \\
$5 \mathrm{~m} / \mathrm{s}$ & $2.55 \mathrm{E}+04$ \\
$46 \mathrm{~mm}^{2} / \mathrm{s}$ & \\
$5 \mathrm{~m} \mathrm{~m} / \mathrm{s}^{2}$ & \\
$92 \mathrm{~mm}^{2} / \mathrm{s}$ & $5.10 \mathrm{E}+04$ \\
$10 \mathrm{~m} / \mathrm{s}$ & \\
$46 \mathrm{~mm}^{2} / \mathrm{s}$ & \\
\hline
\end{tabular}


The increase of pressure amplitude obtained changing the sliding speed or the working temperature of the fluid, permits to reach the cavitation conditions for greater values of the gap, thus improving the bearing performance, with the limitations previously explained.

The influence of the depth of the dimple is rather marginal, because it does not change the pressure profile significantly, as shown in Figure 9.

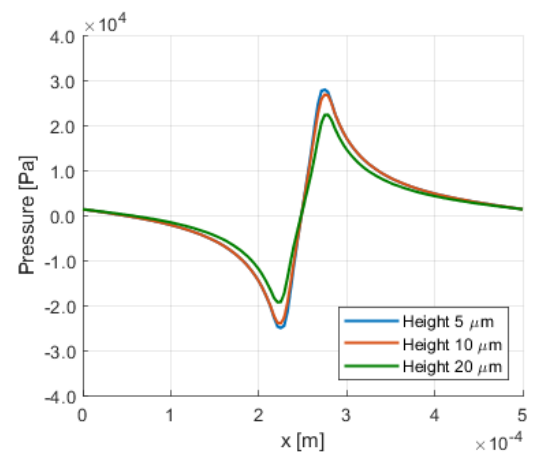

Figure 9: Influence of the dimple depth on the pressure profile. Square dimple with side $50 \mu \mathrm{m}$ and gap $10 \mu \mathrm{m}$, sliding velocity 5 $\mathrm{m} / \mathrm{s}$.

\subsection{Full texturing}

The firsts simulations have demonstrated that with a single dimple it could be possible to obtain a positive load capacity, only for low pressure couplings and when localized cavitation phenomena take place. In order to overcome these limitations further analyses were carried out on a geometry characterized by a sequence of equally spaced dimples over the entire considered domain, Figure 10.

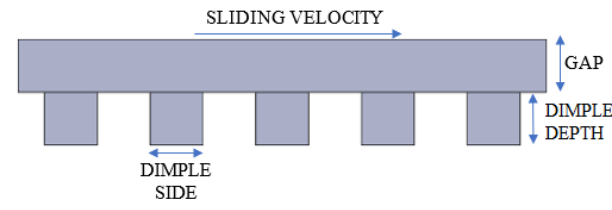

Figure 10: Graphic representation of a full texturing domain with the main geometric features indicated.

In case of the full textured surface the pressure profile trend near a dimple is similar at the single dimple case, already investigated. The pressure field has a periodic oscillation, as can be noted looking at the contour in Figure 11, also represented from the pressure profile calculated along the red line, Figure 12. The calculation of the average pressure value along the central pressure profile confirm that no bearing force is generated.

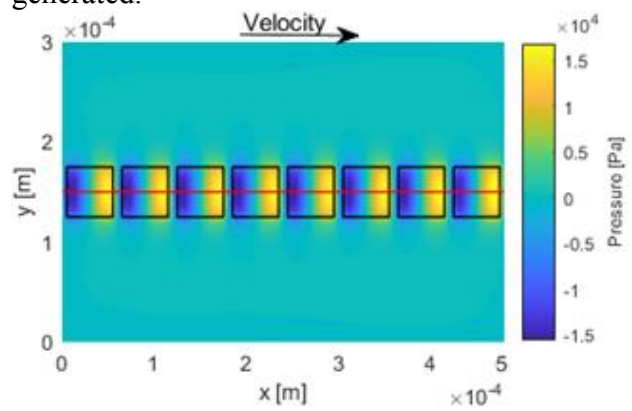

Figure 11: Pressure contour for the case of full texturing. Square dimple with side $50 \mu \mathrm{m}$ and depth $10 \mu \mathrm{m}$. Gap $10 \mu \mathrm{m}$, sliding velocity $5 \mathrm{~m} / \mathrm{s}$.

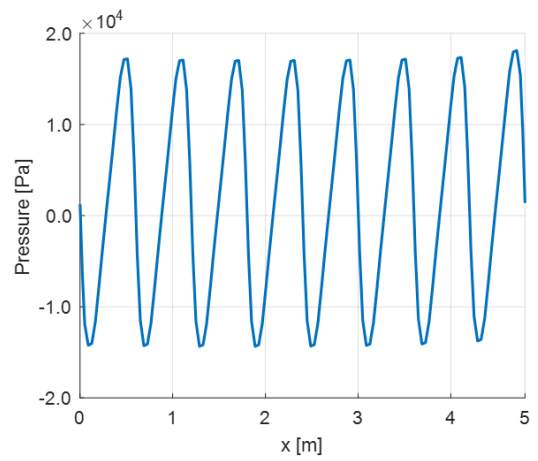

Figure 12: Pressure profile taken from red line of Figure 11. Square dimple with side $50 \mu \mathrm{m}$ and depth $10 \mu \mathrm{m}$. Gap $10 \mu \mathrm{m}$, sliding velocity $5 \mathrm{~m} / \mathrm{s}$.

Therefore, it is clear that in presence of a fully textured domain, it is not possible to obtain any improvement in terms of load capacity, this result being valid regardless of the geometrical dimensions of the dimples. 


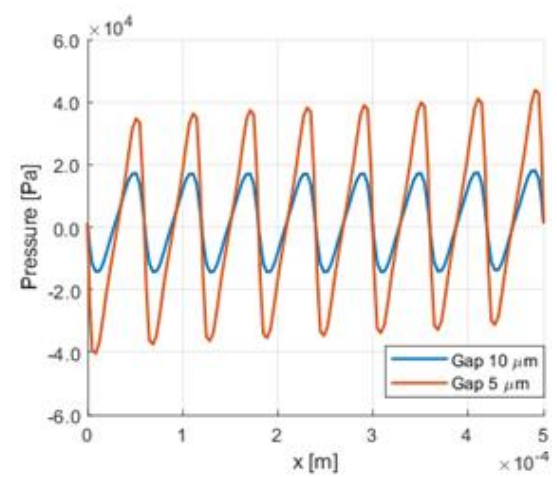

Figure 13: Pressure profile for full texturing, effect of the gap clearance. Square dimples with side $50 \mu \mathrm{m}$ and depth $10 \mu \mathrm{m}$. Sliding velocity $5 \mathrm{~m} / \mathrm{s}$

As for the single dimple case, a reduction of the gap clearance leads to increase the amplitude of the pressure profile; the oscillatory trend remains unchanged not generating any bearing capacity, as shown in Figure 13.

In case of a full textured domain the onset of cavitation appears either when the gap is reduced (as for the single dimple) or also placing the first dimple far from the domain inlet, as shown in Figure 14, where the first dimple has been removed.

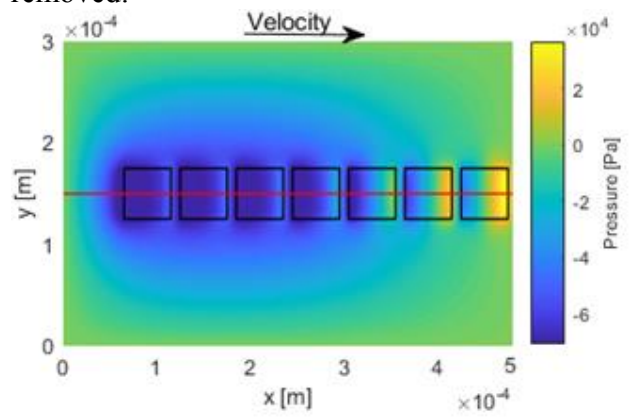

Figure 14: Pressure contour of a full texturing case without the first dimple. Square dimple with side $50 \mu \mathrm{m}$ and depth $10 \mu \mathrm{m}$. Gap 5 $\mu \mathrm{m}$, sliding velocity $5 \mathrm{~m} / \mathrm{s}$.

In this case, as can be seen from the pressure contour, the first dimples show considerable cavitating area, that progressively decreases until it completely ceases. As a consequence of the onset of cavitation the symmetry in the pressure is been distorted. After the firsts dimples the trend is an increasing of the pressure, since each dimple gives a contribution to raise the pressure, but the resulting force on the moving plane is still negative.

As reported in Figure 15, if the clearance is greater, $10 \mu \mathrm{m}$, and the dimples are arranged on the surface in the same way, there is not the formation of cavitation but it is still present an asymmetry of the pressure profile due to the marked depressurization of the first dimples, after that the pressure oscillates symmetrically. Increasing the domain length and the number of dimples the trend does not change and the consequence is an overall force on the moving plane negative.

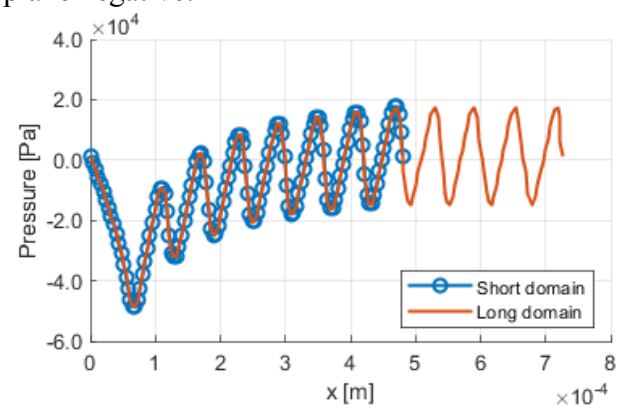

Figure 15: Pressure profile as varying the domain length and the number of dimples. Gap 10 $\mu \mathrm{m}$, sliding velocity $5 \mathrm{~m} / \mathrm{s}$

The effects of the fluid viscosity or the sliding velocity of the moving plane are the same highlighted in the single dimple analysis. In the full textured domain the depth of the dimples affects the pressure profile in a negligible way.

The study of the entire considered domain was carried out considering a single row of dimples; moving to a multi-row configuration, taking care that all the dimples are the same and equally distributed, lead to a condition where all the pressure profiles (at the center of each row) are exactly the same. Figure 16 shows the pressure contour on the moving plane in a generic two rows configuration with indicated two colored lines on each row of dimples. In Figure 17 the pressure profiles along these lines are reported: the pressure perturbations are exactly the same. 


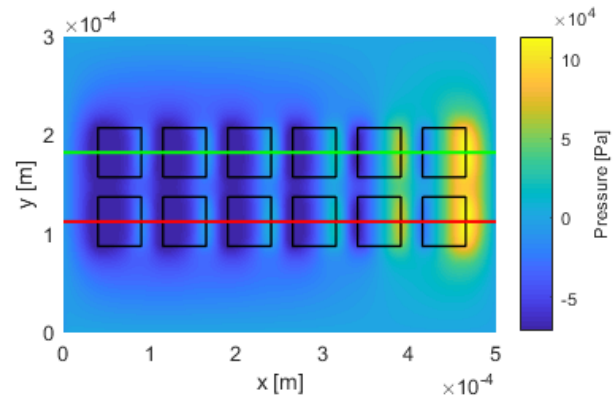

Figure 16: Pressure contour of two row of dimples. Square dimple with side $50 \mu \mathrm{m}$ and depth $10 \mu \mathrm{m}$. Gap $5 \mu \mathrm{m}$, sliding velocity $5 \mathrm{~m} / \mathrm{s}$.

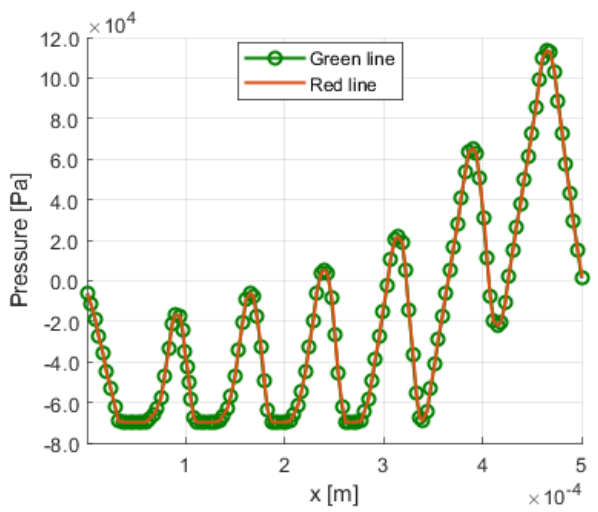

Figure 17: Pressure profiles calculated on the lines shown in the Figure 16.

From the simulations performed, texturing the entire domain does not appear a useful way to increasing the performance of lubricated surfaces coupled in relative motion since the pressure profile oscillates around a zero value and in case of cavitation onset the resulting force is negative.

A positive aspect due to the realization of a fully textured surface is the reduction of the friction coefficient. Since the average fluid film thickness is increased, a full textured surface allows to reduce the viscous friction coefficient: the greater the textured portion of the available surface, the greater the friction coefficient reduction; however, increasing the textured portion means also reduce the coupled surface.

\subsection{Partial texturing}

Starting from the results obtained on both the single dimple and the entire textured domain it is clear that only in a limited number of conditions it is possible to obtain a positive bearing capacity able to counteract against the approaching of the two surfaces in relative motion.

Further investigations are required to obtain a positive load capacity in any working configuration of a lubricated coupling. One possible option is to create a texture of dimples only in a part of the coupled domain. A partial texturing presents relevant improvements in this direction. Partial texturing means to position the dimples only on a portion of the available domain, as shown in Figure 18.

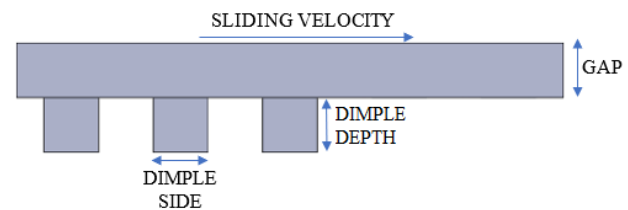

Figure 18: Graphic representation of a partial textured domain with the main geometric features indicated.

Figure 19 shows the pressure contour relating to the case of texturing the $47 \%$ of the entire domain. At the last dimple the pressure rises in a marked way and then gradually fades as can also be appreciated by analyzing the pressure profile.

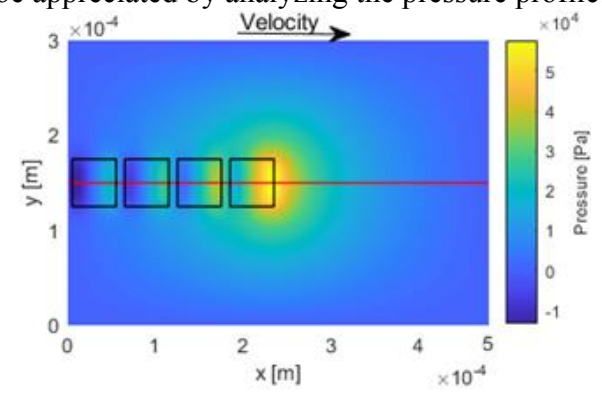

Figure 19: Pressure contour. Square dimple with side $50 \mu \mathrm{m}$ and depth $10 \mu \mathrm{m}$. Gap $10 \mu \mathrm{m}$, sliding velocity $5 \mathrm{~m} / \mathrm{s}$.

In Figure 20 the pressure profiles are shown as a function of textured portion, the pressure does not oscillate symmetrically like the previous cases, but each dimple provides a contribution that raises the pressure locally. The result is a pressure ramp capable of generating a relevant load capacity. 


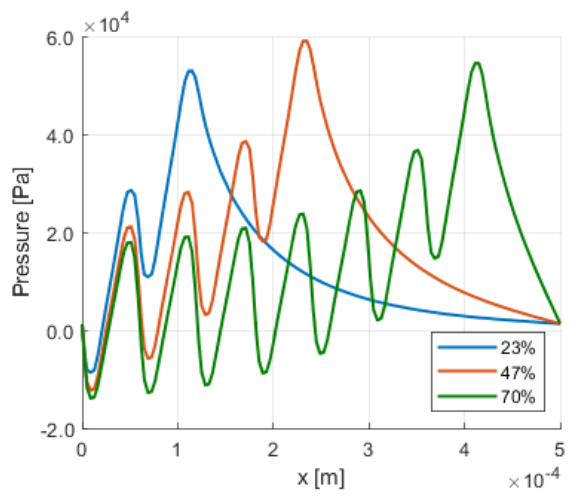

Figure 20: Pressure profile as a function of textured portion. Square dimple with side $50 \mu \mathrm{m}$ and gap $10 \mu \mathrm{m}$, sliding velocity $5 \mathrm{~m} / \mathrm{s}$.

Since in the partial texturing each dimple gives a contribute on locally increase the pressure, it is preferable to have the dimples closest together as possible in order to insert a higher number of it on the same domain, improving the performance of the surface.

In Figure 21 is reported the generated force as a function of the textured portion of the domain. The force values are divided by a reference value for confidential reasons. The best configuration is when the dimples are arranged in the initial 50$60 \%$ of the available domain.

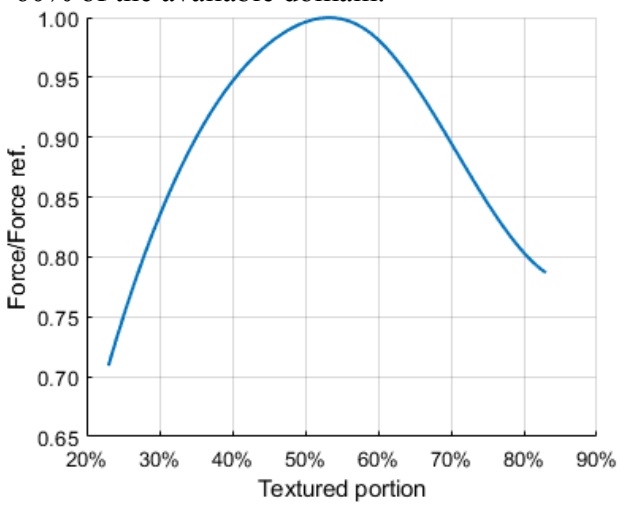

Figure 21: Force as a function of textured portion. Square dimple with side $50 \mu \mathrm{m}$ and gap 10 $\mu \mathrm{m}$, sliding velocity $5 \mathrm{~m} / \mathrm{s}$.

In the partial texturing the position of the first dimple is very important. Increasing the distance from the beginning of the domain means allowing the decrease of the pressure on the first dimples, Figure 22, reducing the peak of over pressure achievable. Moreover, in this configuration the problem of cavitation phenomenon is more pronounced, as the gap decrease. The first dimples are in strong depression, but the partial texturing allows to recover the pressure and guarantee, even in these conditions, a positive bearing capacity.

In order to completely avoid the onset of cavitation it is preferable to place the first dimple exactly at the beginning of the domain with the aim of not creating a divergent zone in the gap.

The reduction of the clearance value amplifies the pressure profile allowing to reach higher values of pressure, Figure 22; the negative aspect is that it also generates smaller values in the area in depression. For this reason it is fundamental to plan the position of the first dimple.

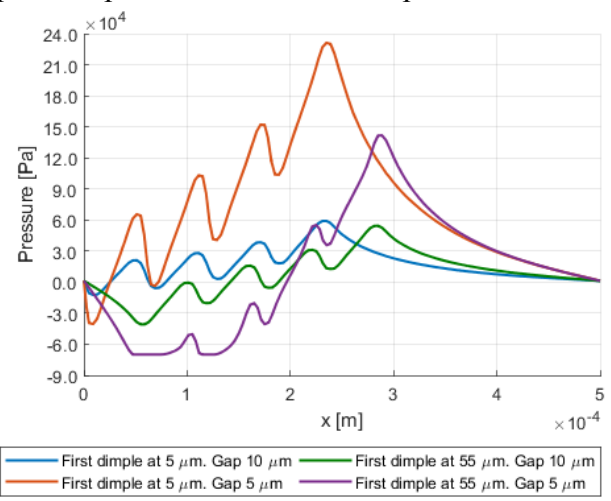

Figure 22: Effect of the disposition of the first dimple and of the gap depth. Textured portion $47 \%$. Sliding velocity $5 \mathrm{~m} / \mathrm{s}$

In partial texturing, the effects of either the fluid viscosity or the sliding velocity of the moving plane are the same highlighted in the previous sections; the depth of the dimples affect the pressure profile in a negligible way also in this case.

A partial textured surface still allows to reduce the friction coefficient between the two moving planes but to a lesser extent than full textured surface since the fluid film thickness is increased only on a portion of the available domain.

Moreover, increasing the number of textured row (equal to each other) on the surface improve the results for a single row.

\section{CONCLUSION AND OUTLOOK}

In this work the preliminary study of a research activity concerning textured surfaces have been presented. The results have highlighted some of the tribological potentials that a textured surface 
could express on a lubricated coupling between sliding surfaces.

The design phase, in terms of dimple geometric definition and spatial distribution, is a challenge due to the high number of variables involved, besides the achievable benefits depend on the operating conditions.

From the CFD simulations conducted it emerged that best solution is to texture only a portion of the available surface in the direction of sliding motion. CFD simulations were carried out considering the cavitation phenomenon, which can significantly influence the bearing performance of the textured surfaces.

Future developments should include a more accurate study of dimples geometry and distribution: the effects due to different dimple shape and orientation have to be analyzed.

Moreover a dynamic analysis of the cavitation phenomenon should be performed. It is worth to investigate how the vapor bubbles are generated and then reabsorbed into the fluid, correlating the characteristic times of the process with the pressure evolution through the dimples.

\section{NOMENCLATURE}

$h \quad$ Gap

$p \quad$ Relative pressure

$U \quad$ Sliding velocity

$\eta \quad$ Dynamic viscosity

\section{ACKNOWLEDGMENTS}

The authors would like to acknowledge the active support of this research by Casappa S.p.A., Parma, Italy.

\section{REFERENCES}

[1] Ibatan T, Uddin M S, Chowdhury M A K, (2015) Recent development on surface texturing in enhancing tribological performance of bearing sliders, Surface \& Coatings Technology, Volume 272, pp 102-120

[2] Dobrica M B, Fillon M, Pascovici M D, Cicone $\mathrm{T}$, (2010) Optimizing surface texture for hydrodynamic lubricated contacts using a massconserving numerical approach, Proceedings of the Institution of Mechanical Engineers Part J Journal of Engineering Tribology, Volume: 224 issue: 8 , pp 737-750
[3] Yu H, Wang X, Zhou F, (2010) Geometric Shape Effects of Surface Texture on the Generation of Hydrodynamic Pressure Between Conformal Contacting Surfaces, Tribology Letters, Volume 37, Issue 2, pp 123-130

[4] Rahmani R, Shirvani A, Shirvani H, (2007) Optimization of Partially Textured Parallel Thrust Bearings with Square-Shaped MicroDimples, Tribology transactions, 50:3, 401-406, DOI: $10.1080 / 10402000701429261$

[5] Kligerman Y, Etsion I, (2001) Analysis of the Hydrodynamic Effects in a Surface Textured Circumferential Gas Seal, Tribology Transactions, Volume 44, Issue 3, pp 472-478

[6] Razzaque M M, Faisal T R, (2008) Performance of Mechanical Face Seals with Surface Micropores, Journal of Mechanical Engineering, Volume 37, pp 77-80

[7] Brizmer V, Kligerman Y, Etsion I, (2003) A Laser Surface Textured Parallel Thrust Bearing, Tribology transactions, 46:3, 397-403, DOI: 10.1080/10402000308982643

[8] Etsion I, Halperin G, Brizmer V, Klingerman Y, (2003) Experimental investigation of laser surface textured parallel thrust bearings, Tribology Letters, Volume 17, pp 295-300

[9] Shah Y G, Vacca A, Dabiri S, (2018) Air Release and Cavitation Modeling with a Lumped Parameter Approach Based on the RayleighPlesset Equation: The Case of an External Gear Pump, Energies, Volume 11, Issue 12, DOI: https://doi.org/10.3390/en11123472

[10] Reid R, Prausnitz J, Poling B, Properties of Gases and Liquids. McGraw-Hill Education, ISBN-13: 978-0070517998 